\title{
Forecasting Future of Manufacturing Systems Driven by Artificial Intelligence: Recent Progress and Future Directions
}

\section{Bahman Zohuri ${ }^{1 *}$ and Farhang Mossavar Rahmani ${ }^{2}$}

${ }^{1}$ Adjunct Professor, Golden Gate University, Ageno School of Business, Data Analytic, San Francisco, California, USA

${ }^{2}$ Professor of Finance and Director of MBA School of Business and Management, National University, San Diego, California, USA

*Corresponding Author: Bahman Zohuri, Adjunct Professor, Golden Gate University, Ageno School of Business, Data Analytic, San Francisco, California, USA
Received: April 03, 2020

Published: April 29, 2020

(C) All rights are reserved by Bahman Zohuri., et al.

\begin{abstract}
The start of a new decade brings a new wave of technological change and unprecedented opportunities that will generate massive amounts of data at the level of Big Data (BD), spawn new threats and transform the way we do business. Dealing with such enormous data structurally or unstructured-wise and process it in real-time requires a new innovative technology such as Artificial Intelligence (AI). Today, AI is capable of learning from its experience through the element of its Machine Learning (ML) in conjunction with the Deep Learning (DL) component and using them to adjust to new input and perform human-like performance, or at least to complement and enhance human abilities. Because of this capability, it encompasses every aspect of the enterprise in the years to come. That is why we believe AI, Automation, and Analytics are central to the success of the enterprise and encompass critical business areas, including data, business processes, the workforce, and risk and reputation.

Keywords: Artificial Intelligence; Machine Learning; Deep Learning; Resilience System; Forecasting and Related Paradigm; Big Data; Fuzzy Logic; Industrial Robotics
\end{abstract}

\section{Introduction}

In recent years, we are witnessing that many industrial organizations and enterprises are rushing toward adopting and implementing Artificial Intelligence (AI) platforms throughout their organizations. This process includes the other elements of AI, namely Machine Learning (ML) and Deep Learning (DL) components, as indicated in figure 1.

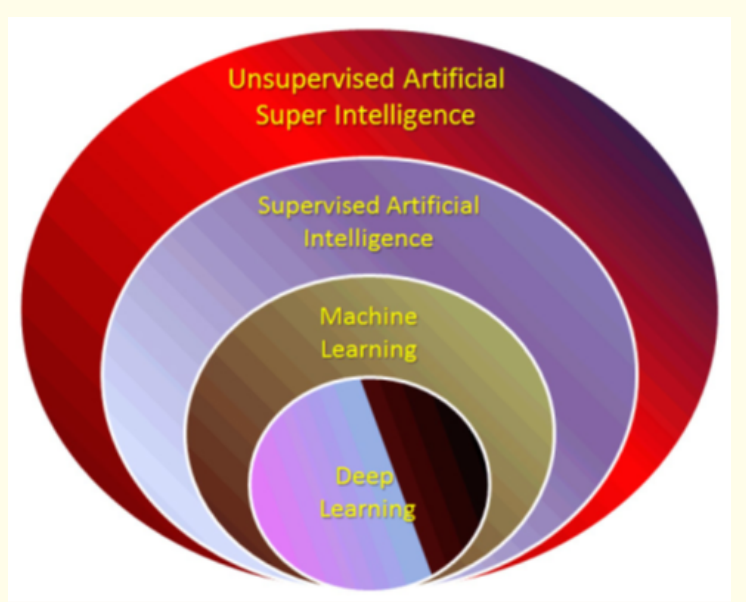

Figure 1: Presentation artificial intelligence in relation with machine learning and deep learning.

Most experts in the field of AI, ML and DL have pooled their insight to discuss how alert organizations can remain one step ahead in the year (s) to come.
In order to stay ahead of the trends with massive shear of coming around by Omni-direction we need some means of prediction or be able to implement some means of forecasting paradigm utilizing appropriate AI platform along with associated ML and DL as integrated elements and learn about $[1,2]$ :

- What the future holds for industries such as 5G, IoT, blockchain and more in a truly connected world with usage of these technologies.

- $\quad$ Proper implementation of AI/ML/DL infrastructure attacks and other crazy-scary things that will keep you up all decade (Figure 2).

- Virtual interface, new ways to work with AI and other factors reshaping the Information Technology (IT) experience.

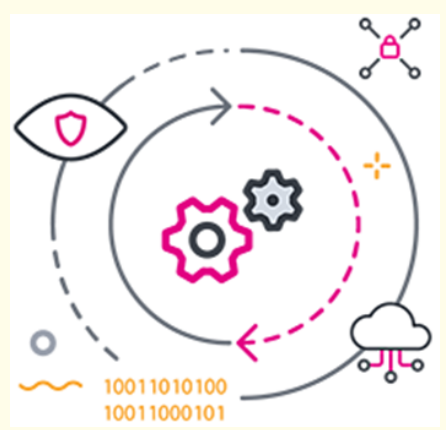

Figure 2: High level industrial IoT. 
Today's Internet of Things (IoT) demonstrates the possibilities and threats that go way beyond the year 2020. In the long-term, for example, one application of IoT and $5 \mathrm{G}$ comes together will be selfdriving cars; thus, we need a paradigm to be able to predict these possibilities and risks [1].

Moreover, Algorithms written around Artificial Intelligence (AI), may decide and take over from its partner human, who gets a job interview and consequently land on that accordingly. This process is a result of that enterprises are going forward via the road of automation and being more robotic oriented. As part of automation and robotic oriented infrastructure, we need a "Build in Feedback" infrastructure, which may be defined as observability of instrument systems and applications to collect matrices and logs by implementing the right algorithms for the proper use case with the industry that your organization is involved with.

Whether a company is involved with a soft aspect of industry such as eCommerce, Banking, Medical, etc., or hard elements of the industry such as sequential line assembly or factory, the robotics implementation is the bread and butter of their process. Because that makes it more efficient and cost-effective, this is where a need for Artificial Intelligence (AI) along with Machine Learning (ML) and Deep Leering (DL) come to play as a significant role for monitoring and observability, as explained: "You can monitor a system using various instrumentation. But if the system doesn't externalize its state well enough that you can figure out what's actually going on in there, then you're stuck".

Moreover, in order to be vigilante and more resilient [2,3], you need digital exhaust time-series data that is based on absorbing these data at the massive layer Big Data (BD). With this massive data in mind, we need help from $\mathrm{AI}$ as a human being so that AI can process incoming data with historical and archive data via its DL and through ML and AI feed the information to its friendly human for ultimate decision making. Given today's massive volume of data at Big Data level, co-operation and interoperability between AI and Human is necessary and sufficient condition that is invadable. This process is another reason that we, as authors, believe, there exist on the separation between humans and AI, yet they are the complement of each other [4].

The day-to-day operations of your Information Technology (IT) systems and applications within your organization all have one thing in common, and that is: They create data as part of their normal operations. Because this data is generated automatically by the networks, storage, mobile devices, and software that run your business, it is classified as Machine Data (MD).

MD has tremendous value. The challenge is finding how to get value from it. Depending on which system, application, or hardware created the data, it can come in a nearly infinite number of formats, ranging from fairly structured data to seemingly random text that is strung together [5].

However, the value of machine data can only be gleaned, or information can be extracted from various sources and processed, particularly in real-time or even near real-time requires augmentation of AI/ML and DL is a necessary element of the IT systems and applications.
By extracting information from Machine Data (MD)and analyzing and correlating it, we learn how to get value from it, which is based on collected historical and present data from the data. Knowing the description of Deep Learning and Machine Learning tasks as infrastructure of Artificial Intelligence, we are not far off from above analysis and correlation of information from Machine Data.

This analysis and correlation, especially at substantial online assembly and manufacturing (i.e. Automobile Assembly Line), allow you as stakeholders to understand how your systems are interoperating the data. This giving you the visibility and information you need to better address outages, reduce mean-time-to-resolution, optimize processes and more.

These are just a few issues that will drive technology conversations and motion toward AI and Automation directions, and every one of them revolves around data and processing these data for the right information [1] if not real-time, but at least near-real-time.

Again, given the nature of this data at the level of sheer volume and massive Big Data (BD) demands AI/ML and DL augmentations.

From AI advances to automation strategies to the new default security framework, organizations that show foresight in 2020 and beyond will be best positioned to turn a challenge into an opportunity [1].

\section{Observability versus monitoring}

Most of the time in industry operation, monitoring and observability are often confused or interchanged, comparing them in table 1 along with figure 3 here, which helps differentiate and define them.

\begin{tabular}{|l|c|}
\hline \multicolumn{1}{|c|}{ Monitoring } & \multicolumn{1}{c|}{ Observability } \\
\hline $\begin{array}{l}\text { Tells you whether the system } \\
\text { works }\end{array}$ & $\begin{array}{c}\text { Lets you ask why it is not work- } \\
\text { ing }\end{array}$ \\
\hline $\begin{array}{l}\text { A collection of metrics and } \\
\text { logs about system }\end{array}$ & $\begin{array}{c}\text { The dissemination of informa- } \\
\text { tion from that system }\end{array}$ \\
\hline Failure-centric & $\begin{array}{c}\text { Understands system behavior } \\
\text { regardless of and outage }\end{array}$ \\
\hline $\begin{array}{l}\text { Is "the how" /Something you } \\
\text { do or make decision around it }\end{array}$ & $\begin{array}{c}\text { Is "the goal"/Something you } \\
\text { have }\end{array}$ \\
\hline I monitor you & You make yourself observable \\
\hline
\end{tabular}

Table 1: Monitoring and observability compresence.

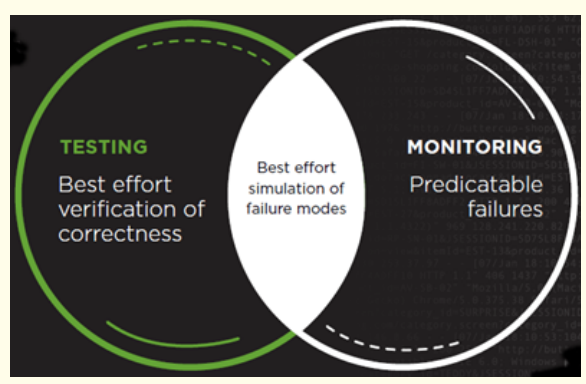

Figure 3: Observability, all possible permutation of full and partial failure. 
“Observability isn't a substitute for monitoring; they're complementary. But it's nearly impossible to have effective monitoring without a culture of observability. Tools are not enough, and none are going to magically "give you" observability".

"If observability is a cultural value, the results will come".

- Andi Mann,

\section{Splunk Chief Technology Advocate.}

Observability as a culture is the degree to which a team or company/organization values the ability to inspect and understand systems, their workload, and their behavior.

Companies and enterprises that have a strong observability culture often have observability teams, although they may not be named as such, for sure dealing with Machine Data/Big Data, they require artificial intelligence and its sub-systems/elements of machine learning, and deep learning, if their use cases need the extracted information or glean it in near real-time if not real-time.

\section{Public key infrastructure (PKI)}

Today more than ever, Information Technology (IT) departments need to understand how digital identities fit into their secure, geographically dispersed architecture. With the workforce moving to remote positions, businesses must concentrate on maximizing productivity through VPN, Wi-Fi and Desktop-as-a-Service access while maintaining a high level of security for remote access. PKI integrated along with AI in any industry or enterprise such as Banking and e-Commerce or other similar organization they are in need of combination of AI and PKI for purpose of Business Continuity (BC) and the Secure Remote Workforce (SRW), when their resource are required or mandated to work remotely.

By definition Public Key Infrastructure (PKI) is a set of roles, policies, hardware, software and procedures needed to create, manage, distribute, use, store and revoke digital certificates and manage public-key encryption. The purpose of a PKI is to facilitate the secure electronic transfer of information for a range of network activities such as e-commerce, internet banking and confidential email. It is required for activities where simple passwords are an inadequate authentication method and more rigorous proof is required to confirm the identity of the parties involved in the communication and to validate the information being transferred as illustrated in figure 4.

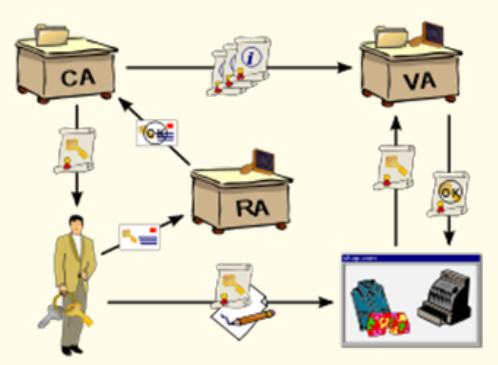

Figure 4: Diagram of a public key infrastructure.
In cryptography, a PKI is an arrangement that binds public keys with respective identities of entities (like people and organizations). The binding is established through a process of registration and issuance of certificates at and by a Certificate Authority (CA). Depending on the assurance level of the binding, this may be carried out by an automated process or under human supervision.

The PKI role that assures valid and correct registration is called a Registration Authority (RA). An RA is responsible for accepting requests for digital certificates and authenticating the entity making the request [6]. In a Microsoft PKI, a registration authority is usually called a subordinate CA [7].

An entity must be uniquely identifiable within each CA domain on the basis of information about that entity. A third-party Validation Authority (VA) can provide this entity information on behalf of the CA.

\section{Conclusion}

Organizations and individual consumers are facing a new wave of technologies that will generate, and allow us to manipulate, untold unique quantities of data. The leaders and technologists in majority companies and enterprises pooled their insights to discuss how this avalanche of data will manifest, and how alert organizations can stay one step ahead.

As it was described before, Artificial Intelligence (AI), along with Machine Learning (ML) and its complementary Deep Learning (DL) is the main driving factor for quotative aspect of the new data, to be able to forecast the future trend of manufacturing systems. This process allows the stakeholders to make smarter yet effective decisions to have better and competitive products in compresence to their rivals in the same market. This action will enable them to be more and more resilient in today's competitive world. An excellent example of such a scenario could be seen in the electronic and digital world, where the smartphone market originally belonged to Blackberry. Later the iPhone took the lead and surpassed the technology of Blackberry, and now the presence of Galaxy Smart Phone has a considerable market share of Apple iPhone.

Being able to forecast future of any manufacturing, either software or hardware aspects of its system and allows the decisionmakers to take the most cost-effective and efficient way of survivability and resiliency for their Organizations, Companies, or Enterprises. Such a forecasting paradigm also enables them to bring the right resource to join their enterprise. This action, in turn, provides the proper growth in their path to the future, with the understanding that $\mathrm{AI}, \mathrm{ML}$, and DL platforms are an invadable scenario.

There exist a massive argument among scholars and other experts that if Artificial Intelligence is a threat to Human Intelligence and someday will push its creator "The Human" and puts him out of business, in particular among "Blue Workers" of any manufactures.

However, these authors argue that AI and humans can go forward by shaking hand and complement each other as much as possible. Of course, we have to look at cases in case situations [4]. 
For example, in banking and retails/eCommerce, where use cases are Application Delivery, Security and Compliance, Business Analytics and Internet of Things need to be enforced and emphasized, augmentation of $\mathrm{AI}$ is a must, given the nature of the beast of the Big Data we are dealing with. In cases like the above, it is very important for a business to be on top of their Customer Relationship Management (CRM), Enterprise Resource Planning (ERP), Individual Customer Retention (ICR) both from rate and strategy perspective and other business applications within the organization, where these use cases are playing huge roles.

Finally, one can explore three areas, where AI can play an enhancement role to its partner Human, and they can be listed here as part of Data Analytics (DA) and Predictive Analytics (PA):

- IT operations,

- IT security, and

- $\quad$ Emerging technologies.

These sorts of observation can predict and allow to look at the near-term synergy of IoT and next generation 5G technologies, and the long-term impact of ignoring user experience. And because leading companies will drive every decision with hard data, we can look at the cost of leaving so much of these data in the dark with continuous publishing series of articles such as this one.

At the end of this conclusion, statistically data shows that $72 \%$ of business leaders believe that AI is going to be a "Business Advantage", according to a study by PwC at www.pwc.com.

Keep in your mind that, Artificial Intelligence (AI) has moved into the mainstream, driven by advances in cloud computing, big data, open source software and improved algorithms. AI technologies are fundamentally altering how we work, live and manage businesses.

\section{Bibliography}

1. B Zohuri and F Mossavar-Rahmani. "A Model to Forecast Future Paradigm, Knowledge Is Power in Four Dimensions". Apple Academic Press, a CRC Press, Taylor and Francis Group (2019).

2. B Zohuri and F Mossavar-Mossavar. "Artificial Intelligence Driven Resiliency with Machine Learning and Deep Learning Components". International Journal of Nanotechnology and Nanomedicine 4.2 (2019): 1-3.

3. Zohuri B and Moghaddam M. "Business Resilience System (BRS): Driven Through Boolean, Fuzzy Logics and Cloud Computation: Real and Near Real Time Analysis and Decision-Making System". $1^{\text {st }}$ edition, Springer Publishing Company (2017).

4. B Zohuri and F Mossavar Rahmani. "Artificial Intelligence Versus Human Intelligence A New Technological Race".

5. Splunk Company internal white paper under title of "Three Companies IR Operations Machine Data, Shake Well Business Problems Solved".
6. "An Overview of Public Key Infrastructures (PKI)". Techotopia (2015).

7. Public Key Infrastructure". MSDN (2015).

\section{Assets from publication with us}

- Prompt Acknowledgement after receiving the article

- Thorough Double blinded peer review

- Rapid Publication

- Issue of Publication Certificate

- High visibility of your Published work

Website: www.actascientific.com/

Submit Article: www.actascientific.com/submission.php Email us: editor@actascientific.com

Contact us: +919182824667 